Suzana Santos Costa

\title{
Modelagem para o Escoamento Transiente Horizontal e Quase Horizontal na Perfuração de Poços de Petróleo
}

Tese apresentada como requisito parcial para obtenção do título de Doutor pelo Programa de Pós-Graduação em Engenharia Civil da PUC-Rio.

Orientadores: Sergio A. B. da Fontoura Sidney Stuckenbruck 


$$
\text { Pontifícia } \begin{array}{r}
U_{\text {niversidade }} \text { C }_{\text {atólólica }} \\
\text { Dio Janeiro }
\end{array}
$$

Suzana Santos Costa

\title{
Modelagem para o Escoamento Transiente Horizontal e Quase- Horizontal na Perfuração de Poços de Petróleo
}

Tese apresentada como requisito parcial para obtenção do título de Doutor pelo Programa de Pós-Graduação em Engenharia Civil da PUC-Rio. Aprovada pela Comissão Examinadora abaixo assinada.

\author{
Prof. Sergio Augusto Barreto da Fontoura \\ Orientador \\ Departamento de Engenharia Civil - PUC-Rio
}

Prof. Sidney Stuckenbruck Co-Orientador Olympus Software Científico Engenharia

Dr. André Leibsohn Martins PETROBRAS

Dr. Wellington Campos

PETROBRAS

Prof. Luiz Fernando Alzuguir Azevedo PUC-Rio

Prof. Celso Romanel Departamento de Engenharia Civil - PUC-Rio

Prof. José Eugênio Leal

Coordenador Setorial do Centro Técnico Científico - PUC-Rio

Rio de Janeiro, 21de Fevereiro de 2006. 
Todos os direitos reservados. É proibida a reprodução total ou parcial do trabalho sem autorização da universidade, da autora e do orientador.

\section{Suzana Santos Costa}

Graduou-se em Engenharia Civil pela UFOP (Universidade Federal de Ouro Preto) em 1998, onde participou de diversas atividades acadêmicas relacionadas à Geotecnia. Obteve seu título de Mestre em Geotecnia pela PUC-Rio em 2001, tendo como tema da dissertação a análise de estabilidade de taludes anisotrópicos. Em seguida, iniciou o doutorado com ênfase em engenharia de petróleo e motivou-se com a o tema de hidráulica de perfuração de poços de petróleo, o que culminou no desenvolvimento desta tese.

Ficha Catalográfica

Costa, Suzana Santos

Modelagem para o escoamento transiente horizontal e quase horizontal na perfuração de poços de petróleo / Suzana Santos Costa ; orientadores: Sergio A. B. da Fontoura, Sidney Stuckenbruck. - Rio de Janeiro : PUC, Departamento de Engenharia Civil, 2006.

145 f. : il. ; $30 \mathrm{~cm}$

Tese (doutorado) - Pontifícia Universidade Católica do Rio de Janeiro, Departamento de Engenharia Civil.

Inclui referências bibliográficas.

1. Engenharia civil - Teses. 2. Hidráulica de poço. 3. Fuxo multifásico. 4. Transiente. 5. Limpeza de poço de petróleo. 6. Pressões durante a perfuração. I. Fontoura, Sergio A. B. da. II. Stuckenbruck, Sidney. III. Pontifícia Universidade Católica do Rio de Janeiro. Departamento de Engenharia Civil. IV. Título. 


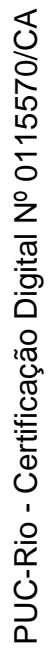

Para Mamãe, Aninha e Rafael 


\section{Agradecimentos}

Aos meus orientadores: Sergio Fontoura, por acreditar em mim e por abrir as portas em um momento difícil, o meu mais sincero obrigado e Sidney Stuckenbruck, pela sua dedicação em me ensinar a Mecânica dos Fluidos, pelas longas conversas sobre hidráulica e sobre a vida, você foi muito importante neste trabalho.

À minha família, especialmente para Mamãe, Aninha, Gabriel, Cíntia e Tia Dodora, obrigado pelo carinho, pelo apoio e pelo amor incondicional e a e "Vó Mary" pelas constantes orações, tenho certeza que iluminaram o meu caminho.

Ao meu namorado Rafael, pelo seu amor, por compartilhar comigo os momentos mais difíceis, por me fazer acreditar a todo o momento que daria certo, você é uma parte muito importante desta conquista. À D. Avany, Maria Cristina e Antônio, pela acolhida nesta família tão cheia de amor!

Aos meus amigos que estiveram presente neste longo caminho, que foram a minha família aqui no Rio: Mércia, Márcia, Talles, Renato, Vanessa e Janaína. Teria sido muito mais difícil sem vocês! Aos amigos que eu fiz ao longo desta longa caminhada: Suzana, Anna Paula, Bruno Holzberg, Ana Lúcia, Flávia Petersen e Ana Paula Alonso.

À André Leibsohn pela amizade, pela confiança e por todo o incentivo.

Aos amigos do GTEP PUC-Rio pela amizade e pelo apoio de vocês. Um obrigado especial a Jorge Pastor, Olga Emília, Shelly Medeiros, Luciana Conceição, Evânia Santos, Camila Borges e Flávio Matos.

Aos funcionários do departamento de Engenharia Civil, principalmente a Ana Roxo, pelos cafés, pelas conversas, pelo carinho com que sempre cuidou dos meus assuntos, fazendo com que se tornassem simples, um obrigado todo especial.

À ANP e ao GTEP PUC - Rio, pelo apoio financeiro. 


\section{Resumo}

Costa, Suzana Santos; Fontoura, Sérgio Augusto Barreto da; Stuckenbruck, Sidney; Modelagem para o escoamento transiente horizontal e quase horizontal na perfuração de poços de petróleo. Rio de Janeiro, 2006. 145 p. Tese de Doutorado - Departamento de Engenharia Civil, Pontifícia Universidade Católica do Rio de Janeiro.

Dentre os custos considerados na explotação de um campo de petróleo, os de perfuração constituem uma parcela significativa do total. Dentro deste cenário, devemos estar atentos à remoção dos cascalhos gerados pela perfuração, também referido como limpeza de poços. Esta operação é, ainda hoje, um tema crítico na perfuração de poços de alta inclinação, pois os cascalhos que se depositam devido à ação da gravidade, formam um leito no interior do espaço anular formado entre a coluna de perfuração e o revestimento. Quando este leito ocupa grande parte do espaço anular, ele é responsável por diversos problemas na perfuração, como por exemplo, desgaste prematuro da broca, baixas taxas de penetração, fraturamento da formação, torques e arrastes excessivos na coluna de perfuração, prisão da coluna de perfuração, interrupção da circulação de fluido, aumento da pressão no anular, etc. Se esta situação não for tratada adequadamente, o problema pode provocar a perda do poço. A tese propõe uma modelagem para o escoamento multifásico na perfuração de poços de petróleo, capaz de avaliar a formação do leito de cascalhos e prever oscilações de pressões no anular decorrentes do escoamento. O modelo adotado é o de duas camadas, onde o espaço anular é dividido em duas regiões: leito e suspensão. O leito (Região 1) é formado pelos cascalhos que se sedimentam devido ao efeito gravitacional enquanto a suspensão (Região 2) é a porção do anular acima do leito depositado, formada pelo fluido de perfuração e os cascalhos transportados. As equações que constituem o modelo implementado são dadas pelas equações de conservação de massa para os sólidos e para o líquido e as equações de conservação de quantidade de movimento para o leito e para a suspensão. O método dos volumes finitos foi utilizado para a discretização das equações diferenciais juntamente com o método de Newton para a solução do sistema não-linear de equações. A solução é dada através das seguintes variáveis: altura do leito, velocidade dos sólidos no leito e na suspensão, velocidade do fluido no leito e na suspensão, 
pressão no anular e concentração de sólidos no anular. Exemplos de aplicação da metodologia são apresentados e mostram o comportamento das variáveis envolvidas ao longo do tempo. Os exemplos mostram a eficácia da metodologia para simular operações de perfuração, em especial, a limpeza de poço.

\section{Palavras-chave}

Hidráulica de poço, fluxo multifásico; transiente, limpeza de poço de petróleo, pressões durante a perfuração. 


\section{Abstract}

Costa, Suzana Santos; Fontoura, Sérgio Augusto Barreto da (Advisor); Stuckenbruck, Sidney (Advisor). Transient modeling of horizontal and near horizontal for wellbore drilling. Rio de Janeiro, 2006. 145 p. Tese de Doutorado - Departamento de Engenharia Civil, Pontifícia Universidade Católica do Rio de Janeiro.

Among the costs considered on an oil field exploration, the drilling process related ones constitute a significant share. Consequently, the focus on the removal of cuttings generated by the drilling process, or the hole cleaning operation, is essential. This operation remains a critical issue in the drilling of high inclination-wells, since the cuttings tend to deposit themselves due to gravity's action, forming a bed in the annular space formed between the drill string and the casing. When this bed takes a sizable fraction of the annular space, it becomes responsible for many problems that appear on the drilling stage, such as premature bit's exhaustion, low rates of penetration, formation fracture, excessive torque and drag on the drillstring, stuck pipe, fluid flow interruption, etc. If this situation is not treated properly, it may cause the loss of the well. This present thesis proposes a model for multiphase flow in the wellbore drilling, capable of evaluating the formation of the cuttings' bed and to predict oscillations of pressures in annulus due to the flow. A two-layer model is adopted, where the annular space is divided in two regions: bed and suspension. The bed (Region 1) is formed by the cuttings, which were deposited due to the action of gravitational force, while the suspension (Region 2) is the portion of the annular above the deposited bed, formed by the drilling fluid and cuttings. The constitutive equations of the aforementioned model are given by the equations of mass conservation for solids and liquids and the momentum conservation equations for the bed and the suspension. The finite volumes method was used to turn the differentials equations into discrete ones, while the Newton's method was applied for the solution of the nonlinear system of equations. The solution is given through the following variables: bed height, solid velocity and fluid velocity both in bed and suspension, annular pressure and solid concentration in the annular. Examples of application of the methodology are presented, showing the behavior of the involved variables through time. The examples show the efficacy of the methodology to simulate drilling operations, in special hole cleaning ones. 


\section{Keywords}

Drilling hydraulic, multiphase flow, transient, hole cleaning, petroleum, pressure while drilling.

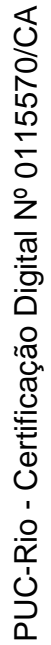




\section{Sumário}

Suzana Santos Costa

1 Introdução 23

1.1. Objetivos 24

1.2. Escopo da tese 25

2 Revisão Bibliográfica $\quad 27$

2.1. Limpeza de poços de petróleo $\quad 29$

2.2. Principais fatores que influenciam na limpeza de poço 30

2.2.1. Velocidade média do fluido no anular 30

2.2.2. Velocidade de sedimentação das partículas 31

2.2.3. Rotação da coluna de perfuração 35

2.2.4. Ângulo de inclinação do poço 35

2.2.5. Propriedades do fluido 36

2.2.6. Tamanho e forma das partículas 38

2.2.7. Excentricidade do anular 38

2.2.8. Taxa de penetração 38

2.3. Modelo reológico $\quad 39$

2.4. Estudos sobre limpeza de poços $\quad 40$

2.5. Padrões de fluxo 43

2.5.1. Fluxo pseudo-homogêneo 43

2.5.2. Fluxo heterogêneo $\quad 44$

2.5.3. Fluxo com leito móvel 44

2.5.4. Fluxo com leito estacionário $\quad 45$

2.6. Modelos de carreamento de sólidos em regime permanente 45

2.6.1. Modelo de duas camadas 48

2.6.2. Modelo de três camadas $\quad 55$

2.6.3. Considerações sobre os modelos de camadas $\quad 59$

$\begin{array}{ll}\text { 2.7. Modelos em Regime Transiente } & 60\end{array}$

3 Modelo Transiente Proposto 62 
3.1. Hipóteses do modelo 62

3.2. Equações Básicas 63

3.2.1. Concentração de Sólidos no Leito 64

3.2.2. Concentração de Sólidos na Suspensão 65

3.3. Equações de Conservação 66

3.3.1. Equação de continuidade para os sólidos 66

3.3.2. Equação de continuidade para o líquido 67

3.3.3. Equação de quantidade de movimento para o leito 68

3.3.4. Equação de quantidade de movimento para a suspensão 70

3.3.5. Tensões Cisalhantes na Parede e Interface 71

3.3.6. Força de Coulomb 72

3.3.7. Condição de Deslizamento (Coulomb) 73

3.4. Deslizamento Sólido-Líquido $\quad 75$

3.4.1. Deslizamento no leito 75

3.4.2. Deslizamento na suspensão 76

4 Discretização e Linearização $\quad 81$

4.1. Equação de conservação de massa para o líquido 82

4.2. Equação de conservação de massa para o sólido + líquido 83

4.3. Equação de quantidade de movimento para a região 1

4.4. Equação de quantidade de movimento para a região 2

4.5. Condições Iniciais 86

4.6. Solução do Modelo

5 Exemplos $\quad 89$

5.1. Formação do leito $\quad 89$

5.2. Erosão do leito 98

5.3. Aumento da taxa de penetração 104

5.4. Aumento da taxa de penetração e da vazão do fluido 107

5.5. Aumento da taxa de penetração e circulação do fluido 110

5.6. Influência da excentricidade da coluna 114

5.7. Influência da inclinação do duto 115

5.8. Influência do deslizamento sólido líquido 116

5.9. Influência dos parâmetros reológicos $\quad 117$

5.10. Influência do fator de atrito nas paredes 119

5.11. Influência do fator de atrito na interface 120 
6 Conclusões e Comentários Finais 122

6.1. Sugestões para futuros trabalhos 124

7 Referências Bibliográficas $\quad 125$

Apêndice A - Coeficientes das Equações de Conservação 130

A.1. Equação de conservação de massa para o líquido 130

A.2. Equação de conservação de massa para o sólido + líquido 131

A.3. Equação de quantidade de movimento para a Região 1

A.4. Equação de quantidade de movimento para a Região 2

Apêndice B - Deslizamento Sólido-Líquido no Leito 134

B.1 As equações de Kozeny (1974) e Ergun (1952) 136

Apêndice C - Relações Geométricas no Círculo 138

$\begin{array}{ll}\text { C.1 Equações básicas } & 138\end{array}$

$\begin{array}{ll}\text { C.2 Caso } 1\left(h<h_{i}\right) & 140\end{array}$

$\begin{array}{ll}\text { C.3 Caso } 2\left(h_{i}<h<h_{s}\right) & 141\end{array}$

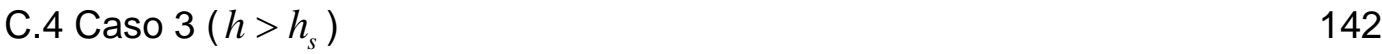

Apêndice D - Adimensionalização das Equações 143 


\section{Lista de figuras}

Figura 2.1 - Transporte de sólidos em trechos verticais. 27

Figura 2.2 - Transporte de sólidos em trechos inclinados e horizontais 28

Figura 2.3 - Esquema do processo de carreamento de cascalhos. 29

Figura 2.4 - Esquema de forças atuando em uma partícula em sedimentação no

interior de um fluido. 32

Figura 2.5 - Variação de velocidade de queda das partículas em função da densidade (Machado, 2001). $\quad 37$

Figura 2.6 - Tipos de excentricidade da coluna de perfuração. 38

Figura 2.7 - Mapa de padrões para o escoamento sólido-líquido. 43

Figura 2.8 - Esquema do Fluxo Pseudo Homogêneo. 44

Figura 2.9 - Esquema do Fluxo Heterogêneo. 44

Figura 2.10 - Esquema do Fluxo com Leito Móvel. 45

Figura 2.11 - Esquema do Fluxo com Leito Estacionário. 45

Figura 2.12 - Esquema do modelo de duas camadas. 48

Figura 2.13 - Ângulo $\theta_{b}$. 53

Figura 2.14 - Regiões do anular. 53

Figura 2.15 - Mapa esquemático do modelo de três camadas. 56

Figura 3.1 - Esquema do escoamento com duas camadas 63

Figura 3.2 - Correlação para a razão entre velocidades. $\quad 79$

Figura 4.1 - Malhas de discretização deslocadas. $\quad 81$

Figura 5.1 - Seção transversal do duto. $\quad 89$

Figura 5.2 - Seção longitudinal do duto. 90

Figura 5.3 - Altura de leito ao longo do comprimento, em intervalos de 10 minutos, para uma vazão de $250 \mathrm{gpm}$ e taxa de penetração de $20 \mathrm{~m} / \mathrm{h} .91$

Figura 5.4 - Altura de leito ao longo do tempo para uma vazão de $250 \mathrm{gpm}$ e taxa de penetração de $20 \mathrm{~m} / \mathrm{h}$ para cinco seções do duto. 92

Figura 5.5 - Altura de leito ao longo do comprimento e do tempo para uma vazão de $250 \mathrm{gpm}$ e taxa de penetração de $20 \mathrm{~m} / \mathrm{h}$. 92

Figura 5.6 - Pressão no anular ao longo do comprimento, em intervalos de 10 minutos, para uma vazão de $250 \mathrm{gpm}$ e taxa de penetração de $20 \mathrm{~m} / \mathrm{h}$. 93

Figura 5.7 - Pressão no anular ao longo do tempo para uma vazão de 250 gpm e taxa de penetração de $20 \mathrm{~m} / \mathrm{h}$ para cinco seções do duto. 
Figura 5.8 - Velocidade do fluido no leito ao longo do comprimento, em intervalos de 10 minutos, para uma vazão de $250 \mathrm{gpm}$ e taxa de penetração de $20 \mathrm{~m} / \mathrm{h}$.

Figura 5.9 - Velocidade dos sólidos no leito ao longo do comprimento, em intervalos de 10 minutos, para uma vazão de $250 \mathrm{gpm}$ e taxa de penetração de $20 \mathrm{~m} / \mathrm{h}$.

Figura 5.10 - Velocidade do líquido no leito ao longo do tempo para uma vazão de $250 \mathrm{gpm}$ e taxa de penetração de $20 \mathrm{~m} / \mathrm{h}$ para cinco seções do duto. 95

Figura 5.11 - Velocidade do fluido na suspensão ao longo do comprimento, em intervalos de 10 minutos, para uma vazão de $250 \mathrm{gpm}$ e taxa de penetração de $20 \mathrm{~m} / \mathrm{h}$.

Figura 5.12 - Velocidade dos sólidos na suspensão ao longo do comprimento, em intervalos de 10 minutos, para uma vazão de $250 \mathrm{gpm}$ e taxa de penetração de $20 \mathrm{~m} / \mathrm{h}$.

Figura 5.13 - Velocidade na suspensão ao longo do tempo para uma vazão de $250 \mathrm{gpm}$ e taxa de penetração de $20 \mathrm{~m} / \mathrm{h}$ para cinco seções do duto.

97

Figura 5.14 - Concentração de sólidos na suspensão, em intervalos de 10 minutos, para uma vazão de $250 \mathrm{gpm}$ e taxa de penetração de $20 \mathrm{~m} / \mathrm{h} . \quad 97$

Figura 5.15 - Altura de leito ao longo do comprimento, em intervalos de 5 minutos, para uma vazão de fluido de $300 \mathrm{gpm}$.

98

Figura 5.16 - Altura de leito ao longo do tempo para uma vazão de fluido de 300 gpm, para cinco seções do duto.

Figura 5.17 - Altura de leito ao longo do tempo para uma vazão de fluido de 300 gpm.

Figura 5.18 - Pressão no anular ao longo do comprimento, em intervalos de 5 minutos, para uma vazão de fluido de $300 \mathrm{gpm}$.

100

Figura 5.19 - Pressão no anular ao longo do tempo para uma vazão de fluido de $300 \mathrm{gpm}$, para cinco seções do duto.

100

Figura 5.20 - Velocidade do líquido no leito ao longo do comprimento, em intervalos de 5 minutos, para uma vazão de fluido de $300 \mathrm{gpm}$.

101

Figura 5.21 - Velocidade dos sólidos no leito ao longo do comprimento, em intervalos de 5 minutos, para uma vazão de fluido de $300 \mathrm{gpm}$.

101

Figura 5.22 - Velocidade do líquido no leito ao longo do tempo para uma vazão de fluido de $300 \mathrm{gpm}$, para cinco seções do duto.

102

Figura 5.23 - Velocidade dos sólidos na suspensão ao longo do comprimento em intervalos de 5 minutos, para uma vazão de fluido de 300 gpm. 102 
Figura 5.24 - Velocidade do líquido na suspensão ao longo do comprimento em intervalos de 5 minutos, para uma vazão de fluido de 300 gpm. 103

Figura 5.25 - Velocidade do líquido na suspensão ao longo do tempo para uma vazão de fluido de $300 \mathrm{gpm}$, para cinco seções do duto.

103

Figura 5.26 - Concentração de sólidos na suspensão em intervalos de 5 minutos, para uma vazão de fluido de $300 \mathrm{gpm}$. 104

Figura 5.27 - Taxa de penetração ao longo do tempo.

104

Figura 5.28 - Altura de leito ao longo do comprimento, em intervalos de 10 minutos, para uma vazão de fluido de $250 \mathrm{gpm}$.

Figura 5.29 - Altura de leito ao longo do tempo para uma vazão de fluido de 250 gpm, para cinco seções do duto. 105

Figura 5.30 - Altura de leito ao longo do comprimento e do tempo. 106

Figura 5.31 - Pressão no anular ao longo do comprimento, em intervalos de 10 minutos, para uma vazão de fluido de $250 \mathrm{gpm}$.

106

Figura 5.32 - Pressão no anular ao longo do tempo para uma vazão de fluido de 250 gpm, para cinco seções do duto.

107

Figura 5.33 - Variação da taxa de penetração e da vazão do fluido ao longo do tempo.

107

Figura 5.34 - Altura de leito ao longo do comprimento, em intervalos de 10 minutos, para taxa de penetração de 10 e 20 m/h e vazão do fluido de 250 e $300 \mathrm{gpm}$.

108

Figura 5.35 - Altura de leito ao longo do tempo para taxa de penetração de 10 e $20 \mathrm{~m} / \mathrm{h}$ e vazão do fluido de 250 e $300 \mathrm{gpm}$ para cinco seções do duto. 108

Figura 5.36 - Altura de leito ao longo do comprimento e do tempo para um aumento da taxa de penetração de 10 para $20 \mathrm{~m} / \mathrm{h}$ e da vazão do fluido de 250 para $350 \mathrm{gpm}$.

109

Figura 5.37 - Pressão no anular ao longo do comprimento, em intervalos de 10 minutos, para taxa de penetração de 10 e $20 \mathrm{~m} / \mathrm{h}$ e vazão do fluido de 250 e $300 \mathrm{gpm}$.

109

Figura 5.38 - Pressão no anular ao longo do tempo para taxa de penetração de 10 e 20 m/h e vazão do fluido de 250 e 300 gpm para cinco seções do duto.

Figura 5.39 - Variação da taxa de penetração ao longo do tempo.

Figura 5.40 - Altura de leito ao longo do comprimento, em intervalos de 10 minutos, para taxa de penetração de variando de 10 a $20 \mathrm{~m} / \mathrm{h}$ e de $20 \mathrm{~m} / \mathrm{h}$ a zero. 
Figura 5.41 - Altura de leito ao longo do tempo variando de 10 a 20 m/h e de 20 $\mathrm{m} / \mathrm{h}$ a zero para cinco seções do duto.

Figura 5.42 - Altura de leito ao longo do comprimento e do tempo, para taxa de penetração de variando de 10 a $20 \mathrm{~m} / \mathrm{h}$ e de $20 \mathrm{~m} / \mathrm{h}$ a zero.

Figura 5.43 - Pressão no anular ao longo do comprimento, em intervalos de 10 minutos, para taxa de penetração de variando de 10 a $20 \mathrm{~m} / \mathrm{h}$ e de $20 \mathrm{~m} / \mathrm{h}$ a zero.

Figura 5.44 - Pressão no anular ao longo do tempo para taxa de penetração variando de 10 a $20 \mathrm{~m} / \mathrm{h}$ e de $20 \mathrm{~m} / \mathrm{h}$ a zero para cinco seções do duto. 113 Figura 5.45 - Influência da excentricidade da coluna na altura do leito. 114

Figura 5.46 - Influência da excentricidade da coluna na pressão. 115

Figura 5.47 - Inclinações do duto. 115

Figura 5.48 - Influência da inclinação do duto na altura do leito. 116

Figura 5.49 - Influência do deslizamento entre as fases na altura do leito. 117

Figura 5.50 - Influência da reologia do fluido na altura do leito. 118

Figura 5.51 - Influência da reologia do fluido na pressão. 118

Figura 5.52 - Influência do fator de atrito nas paredes na altura do leito. 119

Figura 5.53 - Influência do fator de atrito nas paredes na pressão. 120

Figura 5.54 - Influência do fator de atrito na interface na altura do leito. 121

Figura 5.55 - Influência do fator de atrito na interface na pressão. 121

Figura C.1 - Esquema dos ângulos para um anular excêntrico - Caso 1.140

Figura C.2 - Esquema dos ângulos para um anular excêntrico - Caso 2. 141

Figura C.3 - Esquema dos ângulos para um anular excêntrico - Caso 3.142 


\section{Lista de tabelas}

Tabela 2.1 - Correlações para determinação do coeficiente de arraste das partículas para fluidos Newtonianos.

Tabela 2.2 - Correlações para determinação do coeficiente de arraste das partículas para fluidos não-Newtonianos (Modelo de Potência). 34

Tabela 5.1 - Parâmetros utilizados na simulação.

Tabela 5.2 - Parâmetros reológicos considerados.

Tabela B.1 - Comparação dos valores de $\kappa$ e $\kappa_{t}$. 


\section{Lista de Símbolos}

$A_{1}$ área transversal definida pela Região 1 (leito), $\mathrm{m}^{2}$

$A_{2}$ área transversal definida pela Região 2 (suspensão), $\mathrm{m}^{2}$

$A_{L} \quad$ área do leito, $\mathrm{m}^{2}$

$A_{l 1}$ área transversal ocupada por líquido na Região 1, $\mathrm{m}^{2}$

$A_{12}$ área transversal ocupada por líquido na Região 2, $\mathrm{m}^{2}$

$A_{\mathrm{S}} \quad$ área da suspensão, $\mathrm{m}^{2}$

$A_{S} \quad$ área transversal ocupada por sólidos nas Regiões 1 e 2, m²

$A_{l} \quad$ área transversal ocupada por líquido nas Regiões 1 e 2, m²

$A_{S 1} \quad$ área transversal ocupada por sólidos na Região 1, $\mathrm{m}^{2}$

$A_{S_{2}} \quad$ área transversal ocupada por sólidos na Região 2, m²

$A_{M} \quad$ área total, $\mathrm{m}^{2}$

$A_{m l} \quad$ área do leito móvel, $\mathrm{m}^{2}$

$A_{s l} \quad$ área do leito estacionário, $\mathrm{m}^{2}$

$A_{T} \quad$ área transversal livre, $\mathrm{m}^{2}$

$A_{i}^{*} \quad$ área total do cilindro-i, $\mathrm{m}^{2}$

$C_{D} \quad$ coeficiente de arraste

$C_{f 1} \quad$ concentração volumétrica de fluido no leito

$C_{f 2}$ concentração volumétrica de líquido na suspensão

$C_{s 1} \quad$ concentração de sólidos na Região 1

$C_{s 2} \quad$ concentração de sólidos na Região 2

$C_{S} \quad$ concentração da suspensão

$C_{M} \quad$ concentração total

$C_{L} \quad$ concentração do leito.

$C_{m l} \quad$ concentração média de sólidos no leito móvel

$C_{s l} \quad$ concentração média de sólidos no leito estacionário

$d_{p} \quad$ diâmetro da partícula, m 
$D_{i} \quad$ diâmetro do cilindro-i, m

$D_{h} \quad$ diâmetro hidráulico do anular, m

$D_{e} \quad$ diâmetro externo, $\mathrm{m}$

$\frac{d P}{d z} \quad$ perda de carga, $\mathrm{Pa} / \mathrm{m}$

e excentricidade

E força devido ao empuxo, $\mathrm{N}$

$f_{S} \quad$ fator de atrito na suspensão

$f_{l} \quad$ fator de atrito no leito

$f_{i} \quad$ fator de atrito na interface

$F_{m l s l}$ força de atrito entre o leito móvel e o leito estacionário, $\mathrm{N}$

$F_{m l} \quad$ força de atrito entre o leito móvel e a parede, N

$F_{L} \quad$ força de atrito entre o leito e a parede, $\mathrm{N}$

$F_{C} \quad$ força de atrito / unidade de comprimento - sólidos-parede, $\mathrm{N}$

$F_{s} \quad$ força decorrente do contato entre as partículas do leito e as paredes do anular, $\mathrm{N}$

$g$ aceleração da gravidade, $\mathrm{m} / \mathrm{s}^{2}$.

$h \quad$ altura do leito, $\mathrm{m}$

$h_{i} \quad$ altura da base até a linha inferior do cilindro-2, $\mathrm{m}$

$h_{s} \quad$ altura da base até a linha superior do cilindro-2, $\mathrm{m}$

$K \quad$ índice de consistência do fluido, Pa.s ${ }^{n}$

$K_{v} \quad$ constante de von Kárman

$L_{12} \quad$ comprimento da interface, $\mathrm{m}$

$L_{1, \text { ext }} \quad$ comprimento do contato entre o leito e a superfície do poço, $\mathrm{m}$

$L_{1, \text { int }} \quad$ comprimento do contato entre o leito e a superfície do drillpipe, $\mathrm{m}$

$\dot{m}_{s} \quad$ fluxo de massa depositada no leito sólido por unidade de volume, $\mathrm{kg} / \mathrm{s}-\mathrm{m}^{3}$

$\dot{m}_{f} \quad$ fluxo de massa (fonte) para o fluido de perfuração por unidade de volume, $\mathrm{kg} / \mathrm{s}-\mathrm{m}^{3}$

$n \quad$ índice de comportamento reológico do fluido

$P_{e} \quad$ força devido a gravidade, $\mathrm{N}$ 
$P_{i} \quad$ perímetro molhado - interface líquido-líquido, $\mathrm{m}$

$P_{w l} \quad$ perímetro molhado - interface líquido-parede, $\mathrm{m}$

$P_{i j} \quad$ perímetro da interface da região-i com a região-j, m

$q_{S} \quad$ deposição volumétrica / unidade de comprimento dos sólidos, $\mathrm{m}^{2} / \mathrm{s}$

$q_{l}$ deposição volumétrica / unidade de comprimento do líquido, $\mathrm{m}^{2} / \mathrm{s}$

$R_{i} \quad$ raio do cilindro-1 (i= 1,2$), \mathrm{m}$

Re número de Reynolds

$S_{s} \quad$ perímetro da região entre a suspensão e a parede, $m$

$S_{s m l} \quad$ perímetro molhado da interface suspensão - leito móvel, m

$S_{m l s l} \quad$ perímetro molhado do leito móvel - estacionário, $\mathrm{m}$

$S_{L} \quad$ perímetro da região entre o leito e a parede, $\mathrm{m}$

$S_{i} \quad$ perímetro da região entre o líquido de suspensão e o leito, $\mathrm{m}$

$u_{1} \quad$ velocidade in situ na Região 1 (para velocidades iguais), m/s

$u_{2} \quad$ velocidade in situ na Região 2 (para velocidades iguais), m/s

$u_{s 1} \quad$ velocidade in situ dos sólidos na Região 1, m/s

$u_{s 2} \quad$ velocidade in situ dos sólidos na Região 2, m/s

$u_{f 2} \quad$ velocidade do líquido na suspensão, $\mathrm{m} / \mathrm{s}$

$u_{l_{1}} \quad$ velocidade in situ do líquido na Região $1, \mathrm{~m} / \mathrm{s}$

$u_{12} \quad$ velocidade in situ do líquido na Região $2, \mathrm{~m} / \mathrm{s}$

$U_{L} \quad$ velocidade do leito, $\mathrm{m} / \mathrm{s}$

$U_{S} \quad$ velocidade da suspensão, $\mathrm{m} / \mathrm{s}$

$U_{M} \quad$ velocidade total, $\mathrm{m} / \mathrm{s}$

$U_{m l} \quad$ velocidade do leito móvel, $\mathrm{m} / \mathrm{s}$

$U_{s l} \quad$ velocidade do leito estacionário, $\mathrm{m} / \mathrm{s}$

$V_{S} \quad$ volume de sólidos por unidade de comprimento e de tempo, $\mathrm{m}^{2} / \mathrm{s}$

$v_{D E P} \quad$ taxa de deposição volumétrica, $\mathrm{m} / \mathrm{s}$

$v_{E N T} \quad$ taxa de carreamento, $\mathrm{m} / \mathrm{s}$

y coordenada vertical perpendicular ao eixo do poço, $\mathrm{m}$ 
$\alpha$ fração da área da Região 2 (suspensão) para a área total

$\beta_{1} \quad$ ângulo que circunscreve setor que define região-3, rad

$\beta_{2} \quad$ ângulo que circunscreve setor no cilindro-2, rad

$\lambda_{1}$ função de atrito entre o leito e a parede do poço

$\phi^{\prime} \quad$ esfericidade

$\rho_{1} \quad$ densidade do leito, $\mathrm{kg} / \mathrm{m}^{3}$

$\rho_{s} \quad$ densidade do sólido, $\mathrm{kg} / \mathrm{m}^{3}$

$\rho_{f} \quad$ densidade do fluido, $\mathrm{kg} / \mathrm{m}^{3}$

$\rho_{S} \quad$ densidade da suspensão, $\mathrm{kg} / \mathrm{m}^{3}$

$\rho_{L} \quad$ densidade do leito, $\mathrm{kg} / \mathrm{m}^{3}$

$\rho_{p} \quad$ densidade da partícula, $\mathrm{Kg} / \mathrm{m}^{3}$

$\tau_{s} \quad$ tensão cisalhante na suspensão, $\mathrm{N} / \mathrm{m}^{2}$

$\tau_{L} \quad$ tensão cisalhante no leito, $\mathrm{N} / \mathrm{m}^{2}$

$\tau_{i} \quad$ tensão cisalhante na interface sólido-líquido, $\mathrm{N} / \mathrm{m}^{2}$

$\tau_{\text {sml }}$ tensão cisalhante entre a suspensão e o leito móvel, $\mathrm{N} / \mathrm{m}^{2}$

$\tau_{m l s l}$ tensão cisalhante entre o leito móvel e o leito estacionário, $\mathrm{N} / \mathrm{m}^{2}$

$\tau_{\text {sml }}$ tensão cisalhante entre a suspensão e o leito móvel, $\mathrm{N} / \mathrm{m}^{2}$

$\tau_{w} \quad$ tensão cisalhante na interface líquido-parede, $\mathrm{N} / \mathrm{m}^{2}$

$\tau_{b}$ tensão cisalhante de Bagnold (suspensão de sólidos), N/m²

$\tau_{\text {sf }}$ tensão cisalhante entre o sólido e o fluido, $\mathrm{N} / \mathrm{m}^{2}$

$\tau_{s} \quad$ tensão cisalhante entre o sólido e a parede, $\mathrm{N} / \mathrm{m}^{2}$

$\tau_{f s} \quad$ tensão cisalhante entre o líquido e o leito, $\mathrm{N} / \mathrm{m}^{2}$

$\varepsilon \quad$ coeficiente de difusão

$\theta_{b} \quad$ ângulo descrito na Figura 2.13, graus

$\Delta s \quad$ comprimento do trecho, $\mathrm{m}$ 


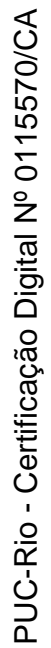

Pedras no caminho? Guardo todas!

Um dia vou construir um castelo!

Fernando Pessoa 